\title{
Joint Interference Detection Method for DSSS Communications Based on the OMP Algorithm and CA-CFAR
}

\author{
Yongshun Zhang , Xin Jia and Ge Song \\ Equipment Academy, 101416 Beijing, China
}

\begin{abstract}
The existing direct sequence spread spectrum (DSSS) communications interference detection algorithms are confined to the high sampling rate. In order to solve this problem, algorithm for DSSS communications interference detection was designed based on compressive sensing (CS). First of all, the orthogonal matching pursuit (OMP) algorithm was applied to the interference detection in DSSS communications, the advantages and weaknesses of the algorithm were analyzed; Secondly, according to the weaknesses of the OMP algorithm, a joint interference detection method based on the OMP algorithm and cell average constant false alarm rate (CA-CFAR) was proposed. The theoretical analyze and computer simulation all proved the effectiveness of the new algorithm. The simulation results show that the new method not only could achieve the interference detection, but also could estimate the interference quantity effectively.
\end{abstract}

\section{Introduction}

Interference detection is an important part of communication anti-jamming technology; it provides the necessary information for the interference suppression. The existed interference detection algorithms could be divided into two parts, one is time-domain interference detection algorithm and the other is transform domain interference detection algorithm. The typical algorithm of time domain interference detection algorithm is the energy detection algorithm [1]. The transform domain interference detection algorithms are mainly based on the different domain characteristics of the signal, background noise and interference. The commonly transform domains include the frequency domain [2], the wavelet domain [3], the fractional Fourier transform domain [4], etc. These methods achieve interference detection successfully, but with the development of technologies, the signal bandwidth is growing, large bandwidth leads to a sharp increase of the sampling rate, the existing methods are confined to the high sampling rate.

Compressive sensing (CS) [5] is a new kind of signal and image processing technology. The technology breaks through the limitation of the Nyquist sampling theorem. This is of great significance in large bandwidth signal processing. At present, the study of signal processing based on CS are mainly focus on the precisely reconstruct of the original signal from compressed signal. However, it is not necessary in many cases, such as signal detection [6]. Due to the structure information of the signal are already included in the compressed signal, we could distinguish whether the interested signal exist just from the compressed data without reconstructing it correctly.
Some scholars proposed the signal detection algorithm based on the CS $[7,8]$.

In this paper, we achieve the DSSS communications interference detection by using the OMP algorithm first. According to the deficiency existing in the algorithm, proposed a joint detection method based on the OMP algorithm and cell average constant false alarm rate (CACFAR). The theoretical basis of the method were analyzed, the effectiveness of the method was verified by computer simulation.

\section{Compressive Sensing}

There are three core issues in CS theory: signal sparse representation, measurement matrix design and signal reconstruction algorithm.

Signal sparse representation: for any signal $\mathbf{s} \in R^{N}$, if there is a transformation matrix $\boldsymbol{\Psi}=\left\{\boldsymbol{\psi}_{1}, \boldsymbol{\psi}_{2}, \boldsymbol{\psi}_{3}, \ldots, \boldsymbol{\psi}_{\mathrm{N}}\right\}$, which satisfies (1), if there only $K(K \ll N)$ on-zero elements in $\boldsymbol{\alpha}$, so $\mathbf{S}$ can be sparse representation in $\boldsymbol{\Psi}$.

$$
\mathbf{s}=\sum_{l=1}^{N} \boldsymbol{\Psi}_{l} \alpha_{l}, \mathbf{s}=\boldsymbol{\Psi} \boldsymbol{\alpha}
$$

Measurement matrix design: we achieve the dimensionality reduction by construction the measurement matrix $\boldsymbol{\Phi}$. The process can be expressed in (2).

$$
\mathbf{y}=\boldsymbol{\Phi s}=\boldsymbol{\Phi} \boldsymbol{\Psi} \alpha=\boldsymbol{\Theta \alpha}
$$

If the signal sparse degree is $K$, the length of the signal is $N$. Then the row numbers of the measurement matrix is $M \geq O\left(K \log _{2}(N / K)\right)$. 
Signal reconstruction: Candès proved that if the (sensing matrix) $\Theta$ satisfies the restricted isometry property (RIP) [9], then we can reconstructed the original signal from the compressed data accurately by solving the optimal problem of $l_{0}$ norm.

$$
\min _{\boldsymbol{\alpha}}\|\boldsymbol{\alpha}\|_{0} \quad \text { s. } \quad \text { t. } \mathbf{y}=\mathbf{\Theta \alpha}
$$

In (3), $\|\bullet\|_{p}$ represents $p-$ norm . Seeking for the sparse representation of a signal from the random sensing matrix is a non-deterministic polynomial problem [10]. A lot of algorithms could be used to solve the problem.

\section{The DSSS communications interference detection BASED on CS}

\subsection{The Problem Description of the Interference Detection in DSSS Communications Based on CS}

In DSSS communications interference detection process, the interested signal is the interference signal. Due to the DSSS signal is similar to the noise, the existence of the DSSS signal does not affect the interference detection seriously. The DSSS communications interference detection problem could be expressed to distinguish the two hypothetical questions as follows.

$$
\begin{gathered}
H_{0}: \quad \mathbf{x}=\mathbf{s}+\mathbf{n} \\
H_{1}: \quad \mathbf{x}=\mathbf{s}+\mathbf{j}+\mathbf{n}
\end{gathered}
$$

In (4) and (5), $\mathbf{s}$ represents the DSSS signal, j represents the interference, $\mathbf{n}$ represents the white Gaussian noise.

If the interference can be sparse representation in $\boldsymbol{\Psi}_{\text {jam }}$, then the above equations could also be expressed in (6) and (7).

$$
\begin{gathered}
H_{0}: \quad \mathbf{x}=\mathbf{s}+\mathbf{n} \\
H_{1}: \quad \mathbf{x}=\Psi_{j a m} \boldsymbol{\alpha}+\mathbf{s}+\mathbf{n}
\end{gathered}
$$

The compressed signal can be expressed as follows.

$$
\begin{gathered}
H_{0}: \mathbf{y}=\boldsymbol{\Phi}(\mathbf{s}+\mathbf{n}) \\
H_{1}: \mathbf{y}=\boldsymbol{\Phi}\left(\Psi_{j a m} \boldsymbol{\alpha}+\mathbf{s}+\mathbf{n}\right)
\end{gathered}
$$

In (9), $\boldsymbol{\alpha}$ represents the coefficient vector, $\Phi$ represents the measurement matrix. From (8) and (9), we know that when the interference does not exist, compressed signal projection values on $\boldsymbol{\Psi}_{\text {jam }}$ is small and distribute randomly. Only when the interference exists, big projection value appears. So we could achieve the interference detection by checking the projection values. Because only a few kinds of interference patterns in DSSS communications system could be sparse representation, we assume that the types of interference may exist in the DSSS communications are known in this paper, so we could get the interference sparse dictionary. This made the interference detection just like signal detection process. So the signal detection method based on the OMP algorithm [8] was used to achieve DSSS communications interference detection firstly.

\subsection{Interference detection algorithm based on the OMP algorithm}

The OMP [8] algorithm is the most commonly used greedy algorithm. Compared to the other reconstruction algorithm, it has low computational complexity. The description of the interference detection algorithm based on the OMP algorithm was as follows.

Input: measurement matrix $\boldsymbol{\Phi}$, interference sparse dictionary $\boldsymbol{\Psi}_{\text {jam }}$, iteration times $K$, threshold $\gamma$;

Initialization: initialization residual $\mathbf{r}_{-} \mathbf{n}=\mathbf{y}$, incremental matrix Aug_t $=[\mathbf{l}$, iterations times time $=1$, coefficient vector aug_y $=[]$;

Output: detection result;

Cycle steps:

Step 1) Search the sensing matrix $\boldsymbol{\Theta}=\boldsymbol{\Phi} \Psi_{\text {jam }}$ for the most relevant column vector with the residual, that is Inp $=\max _{i=1,2 N}\left|\mathbf{r}_{-} \mathbf{n}, \boldsymbol{\psi}_{\mathbf{i}}\right|$;

Step 2) Update the incremental matrix Aug_t $=\left[\right.$ Aug_t, $\left.\boldsymbol{\psi}_{\mathbf{i}}\right]$;

Step 3) Calculate the coefficient vector aug_y by using the least squares method, aug_y $=\left(\text { Aug_t } \mathbf{t}^{\mathrm{T}} * \text { Aug_t }\right)^{(-1)} * \mathbf{A u g} \_\mathbf{t}^{\mathrm{T}} * \mathbf{y}$;

Step 4) Update the residuals r_n = y-Aug_t*aug_y ;

Step 5) If time $<K$, time $=$ time +1 , then return to step 1), else, execute step 6);

Step 6) If $\|$ aug_y $\mathbf{y} \|_{\infty}>\gamma$, the interference exists, else, the interference does not exist.

\section{The joint interference detection method based on the OMP and CA- CFAR}

There three weaknesses in the interference detection algorithm based on the OMP algorithm.

(1) It is difficult to choose the iteration times in the algorithm, especially in the interference detection process;

(2) Due to the threshold changes with the signal-tonoise ratio (SNR), it is difficult to choose the right threshold in the algorithm.

(3) The hardware implemented of the OMP algorithm is still under study $[11,12]$.

In order to overcome the weakness of the algorithm based on the OMP algorithm, we proposed a joint interference detection algorithm based on the OMP algorithm and CA-CFAR.

\subsection{Theoretical analyze of the new algorithm}

The first step of the OMP algorithm is inner product searching. It was show in (10) and (11)

$$
\begin{gathered}
\hat{\mathbf{x}}=\left(\boldsymbol{\Phi} \boldsymbol{\Psi}_{\text {jam }}\right)^{H} *\left(\boldsymbol{\Phi} \boldsymbol{\Psi}_{\text {jam }} \boldsymbol{\alpha}\right) \\
\hat{\mathbf{x}}=\boldsymbol{\Psi}_{\text {jam }}{ }^{H} \boldsymbol{\Phi}^{H} * \boldsymbol{\Phi} * \boldsymbol{\Psi}_{\text {jam }} * \boldsymbol{\alpha}
\end{gathered}
$$

The above expression can be expressed as follows.

$$
\begin{gathered}
\hat{\mathbf{x}}=\boldsymbol{\Psi}_{j a m}{ }^{H} *\left(\boldsymbol{\Phi}^{H} * \boldsymbol{\Phi}\right) * \boldsymbol{\Psi}_{j a m} * \boldsymbol{\alpha} \\
\hat{\mathbf{x}}=\boldsymbol{\Psi}_{j a m}{ }^{H} * \mathbf{P h i} * \boldsymbol{\Psi}_{j a m} * \boldsymbol{\alpha}
\end{gathered}
$$


In (13), Phi is a normal matrix. As can be seen from the above equation, after the calculation, we got avector. Due to the characteristics of the matrix Phi and $\boldsymbol{\Psi}_{\text {jam }}$, $\hat{\mathbf{x}}$ is associated with the coefficient $\boldsymbol{\alpha}$. When there is a big sparse coefficient in $\boldsymbol{\alpha}$, there is a corresponding peak in $\hat{\mathbf{x}}$, so we also could achieve the interference detection by detecting the peak value in $\hat{\mathbf{x}}$. After the above operation, the random characteristics of the compressed data are overcome, we convert the compressed data to the same length as the original signal, the new signal contains the interference signal information, so the conventional detection method will suitable for the interference detection.

CA-CFAR [13] is a common method in radar signal detection. For a given expected average false alarm rate, the required threshold could be obtained by solving the following equations.

$$
\begin{gathered}
\bar{P}_{f a}=\left(1+\frac{T}{L}\right)^{-L} \\
T=L\left(\bar{P}_{f a}^{-1 / L}-1\right)
\end{gathered}
$$

In (14) and (15), $\bar{P}_{f a}$ represents the false alarm rate, $T$ represents the threshold product factor, $L$ represents the length of the lead and lag cell. The final detection threshold was showed as follows.

$$
\hat{T}_{h}=\frac{T}{L} \sum_{i=1}^{L} \hat{\mathbf{x}}_{i}
$$

In (16), $\hat{T}_{h}$ represents the threshold. When the peak value in is greater than the threshold, we believe that the existence of interference. When the peak is less than the threshold, we believe that there is no interference.

\subsection{Algorithm description}

According to the above description of the principle, the proposed algorithm could be described as follows:

Input: measurement matrix $\boldsymbol{\Phi}$, interference sparse dictionary $\boldsymbol{\Psi}_{j a m}$, the false alarm rate $P_{f a}$, the lead and lag cells length $L_{c}$, the guard cell length $L_{g}$;

Initialization: initialization residual $\mathbf{r}_{-} \mathbf{n}=\mathbf{y}$;

Output: detection result.

Steps:

Step 1) Calculate the product of the sensing matrix $\boldsymbol{\Theta}=\boldsymbol{\Phi} \boldsymbol{\Psi}_{\text {jam }}$ and the residual, namely $\hat{\mathbf{x}}=\left(\boldsymbol{\Phi} \boldsymbol{\Psi}_{j a m}\right)^{H} * \mathbf{r} \_\mathbf{n}=\boldsymbol{\Theta}^{H} * \mathbf{r} \_\mathbf{n}$;

Step 2) Using the matched filter to deal with the $\hat{\mathbf{x}}$, output signal is $\mathbf{r}, \mathbf{r}=\hat{\mathbf{x}} * \overline{\hat{\mathbf{x}}}$;

Step 3) Using the CA-CFAR detection method to deal with the output signal of the matched filter, we got to know whether the interference exist using the detection result.

\section{Simulation Results and Analysis}

We take the DSSS communications multi-tone interference detection as an example to verify the effectiveness of the algorithm. The DSSS signal parameters were set as follows: source rate was $100 \mathrm{~kb} / \mathrm{s}$,
PN code was $m$ sequence of 63 , PN code rate was $6.3 \mathrm{Mb} / \mathrm{s}$. The modulation scheme is binary phase shift keying (BPSK), the carrier frequency was $6.3 \mathrm{MHz}$. The multi-tone interference parameters were set as follows: the multi-tone interference frequency was $[5,8] \mathrm{MHz}$, the unit is Hz. The sparse dictionary of the interference is Fourier orthogonal matrix. The CA-CFAR detection algorithm parameters were set as follows: the lead and lag cell is 50 , the guard cell is 2 . We use the Gaussian random matrix as the measurement matrix. The compression ratio is defined as $M=$ actual sampling number/Nyquist sampling number The compression ratio is $1 / 2$ in this paper. Doing 2000 Monte-Carlo simulation for every experiment.

Fig. 1 shows the success rate of interference detection algorithm based on the OMP algorithm in the condition of $10 \mathrm{~dB}$ noise, the threshold was set to 4.9 which was the optimal threshold obtained by multiple simulations, algorithm iteration number was set to 2 . As can be seen from the figure, the algorithm could achieve the interference detection effectively. When the jammer-tosignal ratio (JSR) is more than $-6 \mathrm{~dB}$, the success rate of interference detection was more than 0.95 . This proves that with the prior information of the interference types and the number of iterations, the interference detection algorithm based on the OMP algorithm is feasible for the DSSS interference detection.

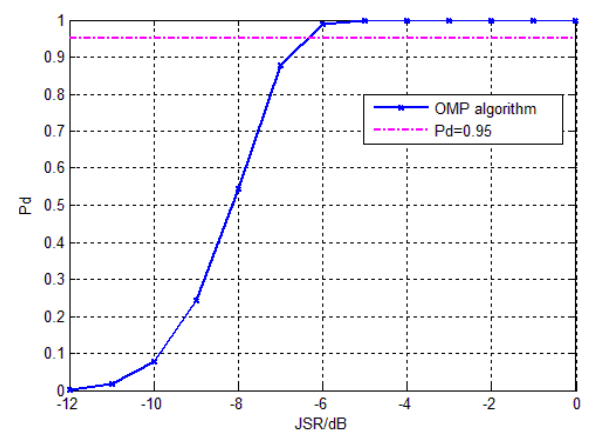

Figure 1. Success rate of interference detection algorithm based on the OMP algorithm (10dB noise)

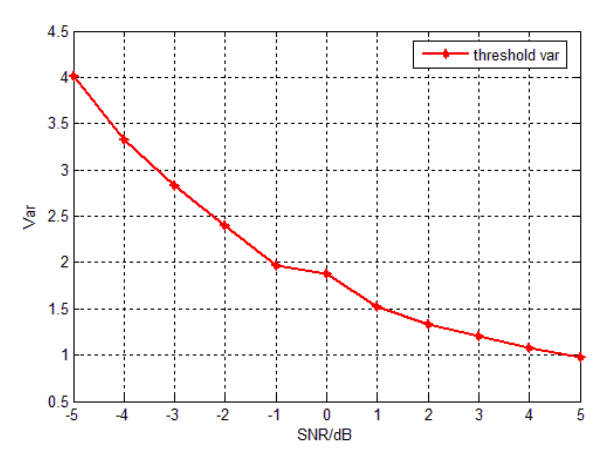

Figure 2. The threshold fluctuation with SNR

In the interference detection algorithm based on the OMP algorithm, the threshold is very important. Fig. 2 shows the threshold fluctuations of the algorithm. As can be seen from the figure, the threshold changes with the SNR, the fluctuations of the threshold is serious, this factors will confine the application of the algorithm. At 
this point, the interference detection algorithm based on the OMP algorithm was not applicable.

Fig. 3 shows the interference detection result using the interference detection algorithm based on the OMP algorithm and CA-CFAR. The JSR was set to $-9 \mathrm{~dB}$, the SNR was set to $10 \mathrm{~dB}$, the false alarm rate was set to $10 \mathrm{e}-$ 6. As can be seen from the figure, the algorithm works correctly in detecting the interference even through the interference power is lower than the signal. It proved that the algorithm is suitable for interference detection in DSSS communications.

CA-CFAR is an adaptive threshold detection algorithm, so the interference detection algorithm based on the OMP algorithm and CA-CFAR is essentially an adaptive threshold interference detection algorithm, the performance of the algorithm does not change over the threshold. Fig. 4 shows the performance of the algorithm based on the OMP algorithm and CA-CFAR in the condition of different SNR, the JSR was set to $10 \mathrm{~dB}$, the false alarm rate was set to $10 \mathrm{e}-6$. As can be seen from the figure, the algorithm works pretty good. The simulation result proved the above analysis.

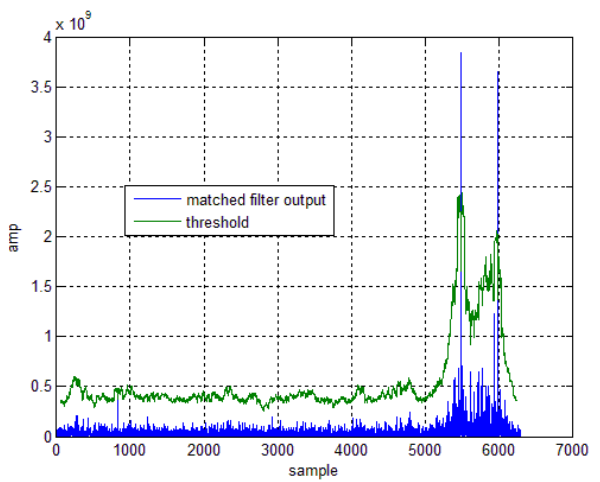

Figure 3. Interference detection result of the algorithm based on the OMP algorithm and CA-CFAR

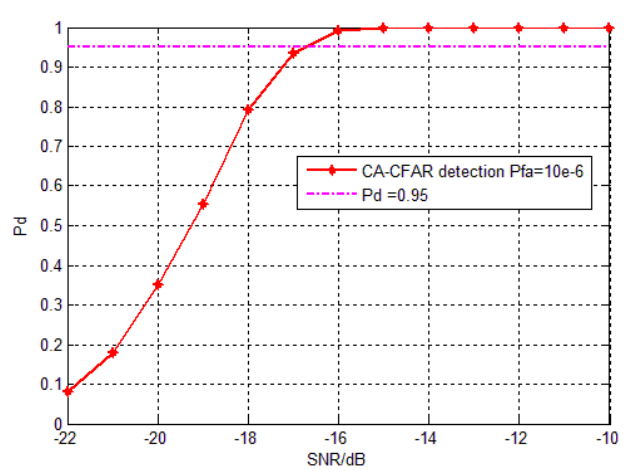

Figure 4. Success rate of interference detection in the condition of different SNR

Fig. 5 shows the comparison of the two algorithms. As can be seen from the figure, the algorithm based on the OMP algorithm and CA-CFAR got the similar performance with the algorithm based on the OMP algorithm when the false alarm rate was set to $10 \mathrm{e}-6$. We also can see that the success rate of the interference detection in the new algorithm changes with the false alarm rate, with a higher success rate of interference detection, the false alarm rate also increased, so there should be a tradeoff between the false alarm rate and success rate of the interference detection in practical.

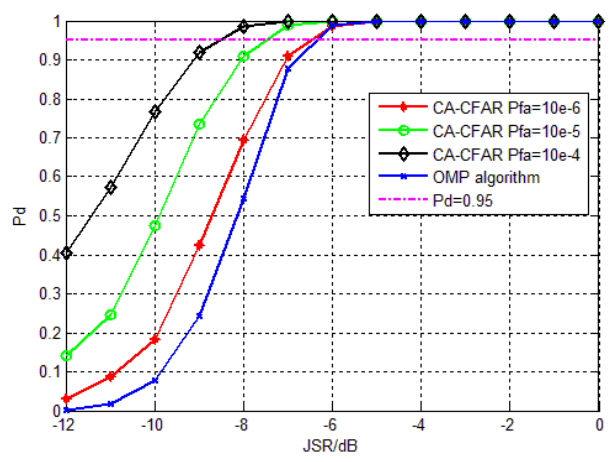

Figure 5. Success rate of the interference detection of the two algorithms

The interference detection algorithm based on the OMP algorithm and CA-CFAR not only can achieve the interference detection, but also can get the quantity information of the interference. Fig. 6 shows the quantity estimation results using the algorithm. As can be seen from the figure, when JSR was more than $6 \mathrm{~dB}$, the estimation result of the interference is close to the real value. According to Fig. 3, we can also see that, when the interference signal index positions in the sparse dictionary were in the same reference unit range, we may not estimate the quantity of the interference accurately.

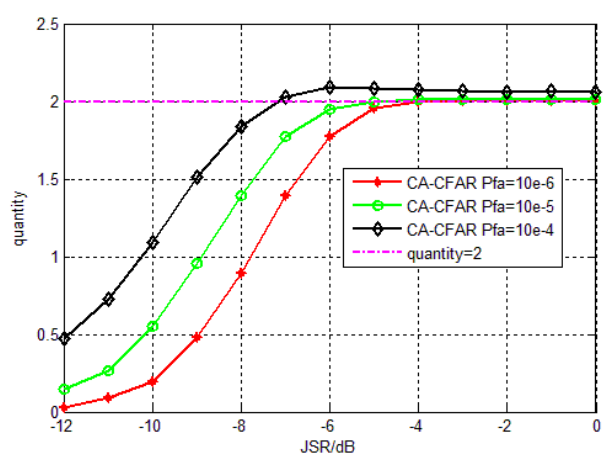

Figure 6. The estimation quantity of the interference

\section{Conclusion}

We proposed the joint compressive sensing interference detection algorithm for the DSSS communications based the OMP algorithm and CACFAR in this paper. With the prior information of the interference types that may exist in the DSSS communications system, the algorithm could achieve the interference detection effectively and it has a good robustness. Due to the algorithm achieves the DSSS communications interference detection by using the CS, the high sampling rate problem in the traditional interference detection algorithms was solved, also the new algorithm is useful to reduce the system costs. Interference detection process is the basis of the interference suppression method, this algorithm will help to achieve the DSSS communication interference suppression in compressed domain and we will carry out the research in this field in the future. 


\section{References}

1. Y. Lee, D. Kang, and H. Park, Proc. 3rd International Conference on Anti-counterfeiting, Security, and Identification in Communication. (2009).

2. T. Yamamoto, K. Takeda, and F. Adachi, Proc. 2009 IEEEE 70th Vehicular Technology Conference Fall. (2009)

3. S. C. Olhed and G. Metikas IEEE Transactions on Signal Processing, 57. (2009).

4. Y. Zhang, Q. Zhang, and S. Wu, 2008 International Conference on International Technology and Applications in Biomedicine. (2008).

5. X. Chu, M. C. Stamm, K. J. R. Liu IEEE Transactions on Information Forensics and Security, 10. (2015).

6. J. Haupt and R. Nowak IEEE International Conference on Acoustics, Speech and Signal Processing. (2007).
7. M. F. Duarte, M. A. Davenport, M. B. Wakin. IEEE International Conference on Acoustics, Speech and Signal Processing. (2006).

8. L. Bing, F. Ping, M. Shengwei, Chinese journal of scientific instrument, 31. (2010).

9. J. Castorena and C. D. Creusere, IEEE Transactions on Signal Processing, 62. (2014).

10. A. Majumdar, R. K. Ward, IEEE Jounal on Emerging and Selected Topics in Circuits and Systems, 2. (2012).

11. F. Lingzhi, M.S. thesis, South-Central University for Nationalities, Wuhan, (2013).

12. X. Chengyi and D. Panfeng, Journal of SouthCentral University for Nationalities (Nat. Sci. Edition), 32. (2012).

13. M. A. Richards. New York: McGraw-Hill. (2005) 